Vol. XX (XXXX)

No. X

\title{
DE DONDER-WEYL EQUATIONS AND MULTISYMPLECTIC GEOMETRY
}

\author{
Cornelius Paufler* and Hartmann Römer ${ }^{\dagger}$ \\ Fakultät für Physik \\ Albert-Ludwigs-Universität Freiburg im Breisgau \\ Hermann-Herder-Straße 3 \\ D 79104 Freiburg i. Br.
}

(Received

2001)

\begin{abstract}
Multisymplectic geometry is an adequate formalism to geometrically describe first order classical field theories. The De Donder-Weyl equations are treated in the framework of multisymplectic geometry, solutions are identified as integral manifolds of Hamiltonean multivectorfields.

In contrast to mechanics, solutions cannot be described by points in the multisymplectic phase space. Foliations of the configuration space by solutions and a multisymplectic version of Hamilton-Jacobi theory are also discussed.
\end{abstract}

Key words: Geometric field theory, Multisymplectic geometry, Hamiltonian formulation

The field of multisymplectic geometry has experienced a revival of active research recently with the discovery of super Poisson-Lie brackets on the canonically arising multisymplectic phase spaces (河, [3). It provides a geometrical framework to formulate classical field theory in a coordinate free manner on arbitrary space-time manifolds. For a detailed review we refer to the excellent exposition (顿), here we only sketch the properties needed for our work.

Consider a classical field $\varphi^{i}(x),(i=1, \ldots, N)$ over $n$-dimensional space-time with a Lagrangean density $\mathcal{L}\left(x^{\mu}, \varphi^{i}, \partial_{\mu} \varphi^{i}\right)$. The corresponding equations of motion are

$$
\frac{\partial \mathcal{L}}{\partial \varphi^{i}}-\partial_{\mu} \frac{\partial \mathcal{L}}{\partial\left(\partial_{\mu} \varphi^{i}\right)}=0
$$

The main idea of the multisymplectic formulation of classical field theory consists in

\footnotetext{
* e-mail: paufler@physik.uni-freiburg.de.

$\dagger$ Talk given by H. R. at the 33rd Symposium on Mathematical Physics, Toruń, Poland, June 2001. e-mail: roemer@physik.uni-freiburg.de.
} 
associating $n$ polymomenta

$$
\pi_{i}^{\mu}=\frac{\partial \mathcal{L}}{\partial\left(\partial_{\mu} \varphi^{i}\right)}, \quad(\mu=1, \ldots, n ; i=1, \ldots, N)
$$

to every component of the field as opposed to the conventional canonical approach, where only $\pi_{i}^{0}=\pi_{i}$ is considered.

The multisymplectic phase space $\mathcal{P}$, thus arising, has dimension $(N+1)(n+1)$ and can be described by local coordinates

$$
\left(x^{\mu}, q^{i}, p_{i}^{\mu}, p\right),
$$

where $x^{\mu}$ correspond to space-time, $q^{i}$ and $p_{i}^{\mu}$ to the values of the field and its polymomenta, respectively. The importance of the additional coordinate $p$, necessary for consistency, will be explained in a moment.

If we assume that the Lagrangean $\mathcal{L}$ be non singular, such that (2) can be inverted and the field derivatives $\partial_{\mu} \varphi^{i}$ can be expressed as functions of $x^{\mu}, \varphi^{i}$, and $\pi_{i}^{\mu}$, then we can define a De Donder-Weyl Hamiltonean

$$
\mathcal{H}\left(x^{\mu}, \varphi^{i}, \pi_{i}^{\mu}\right)=\pi_{i}^{\mu} \partial_{\mu} \varphi^{i}-\mathcal{L}
$$

and we readily see that the equations of motion (1D) are equivalent to the De Donder-Weyl equations

$$
\frac{\partial \mathcal{H}}{\partial \varphi^{i}}=-\partial_{\mu} \pi_{i}^{\mu} ; \quad \frac{\partial \mathcal{H}}{\partial \pi_{i}^{\mu}}=\partial_{\mu} \varphi^{i}
$$

These equations also follow from the variational principle

$$
\delta \int d^{n} x\left(\pi_{i}^{\mu} \partial_{\mu} \varphi^{i}-\mathcal{H}\right)=\delta \int \Theta \stackrel{!}{=} 0
$$

where the variation has to be performed in $\varphi$ and $\pi$ independently.

In a geometric formulation, the fields are sections of a bundle $\mathcal{E} \stackrel{\pi}{\rightarrow} \mathcal{M}$ over an $n$ dimensional space-time manifold $\mathcal{M}$ with typical fibre $\mathcal{Q}$.

The first jet bundle (for an introduction we refer to Saunder's book [11]) of sections of $\mathcal{E}$, denoted $\mathfrak{J}_{1}(\mathcal{E})$, is an affine bundle over $\mathcal{E}$, its dual $\mathcal{P}=\mathfrak{J}_{1}^{*}(\mathcal{E})$ consisting of all affine maps into the line bundle $\Lambda^{n} T M$ is a vector bundle over $\mathcal{E}$. The fibre dimension of $\mathcal{P}$ equals the fibre dimension of $\mathfrak{J}_{1}(\mathcal{E})$ plus 1 , thus accommodating the translation part of the affine maps. The coordinate $p$, mentioned above, is associated to these translations. $\mathcal{P}$ is just the multisymplectic phase space. The correspondences between classical mechanics and multisymplectic classical field theory are collected in the following table. 


\begin{tabular}{|c|c|}
\hline Mechanics $(n=1)$ & Field theory \\
\hline $\begin{array}{l}\text { Extended configuration space } \\
\qquad \mathcal{Q} \times \mathbb{R}\end{array}$ & $\begin{array}{l}\text { Bundle over an } n \text {-dimensional space- } \\
\text { time manifold } \mathcal{M} \text { with typical fibre } Q \\
\qquad \mathcal{E} \stackrel{\pi}{\rightarrow} \mathcal{M}\end{array}$ \\
\hline $\begin{array}{l}\text { Double-extended phase space } \\
\qquad \mathcal{P}=T^{*}(\mathcal{Q} \times \mathbb{R})\end{array}$ & $\begin{array}{l}\text { Multisymplectic phase space, affine } \\
\text { dual of jet bundle } \\
\qquad \mathcal{P}=\mathfrak{J}_{1}^{*}(\mathcal{E})\end{array}$ \\
\hline $\begin{array}{r}\text { Coordinates of } \mathcal{Q} \times \mathbb{R} \\
t, q^{i}\end{array}$ & $\begin{array}{l}\text { Coordinates of } \mathcal{E} \\
\qquad x_{\mu}, q^{i}\end{array}$ \\
\hline $\begin{array}{c}\text { Coordinates of } T^{*} \mathcal{Q} \times \mathbb{R}^{2}=\mathcal{P} \\
t, q^{i}, E, p_{i}\end{array}$ & $\begin{array}{r}\text { Coordinates of } \mathcal{P}=\mathfrak{J}_{1}^{*}(\mathcal{E}) \\
x^{\mu}, q^{i}, p, p_{i}^{\mu}\end{array}$ \\
\hline $\begin{array}{l}\text { Poincaré-Cartan 1-form on } \mathcal{P} \\
\qquad \Theta=p_{i} d q^{i}-E d t\end{array}$ & $\begin{array}{l}\text { Poincaré-Cartan } n \text {-form on } \mathcal{P}, \quad 2 \text { - } \\
\text { horizontal }^{1} \\
\qquad \Theta=p_{i}^{\mu} d q^{i} \wedge d^{n} x_{\mu}-p d t \wedge d^{n} x\end{array}$ \\
\hline $\begin{array}{l}\begin{array}{l}\text { Symplectic } 2 \text {-form on } \mathcal{P}, \\
\text { degenerate }\end{array} \\
\qquad \omega=-d \Theta=d q^{i} \wedge d p_{i}+d E \wedge d t\end{array}$ & $\begin{array}{l}\text { Multisymplectic }(n+1) \text {-form on } \mathcal{P}, \text { non- } \\
\text { degenerate (on vectorfields) } \\
\omega=-d \Theta=d q^{i} \wedge d p_{i}^{\mu} \wedge d^{n} x_{\mu}+d p \wedge d^{n} x\end{array}$ \\
\hline
\end{tabular}

Here, $d^{n} x=d x^{1} \wedge \cdots \wedge d x^{n}$ is the volume form on $\mathcal{M}$, and $d^{n} x_{\mu}=i_{\partial_{\mu}} d^{n} x$.

Some remarks are in order here.

1. Although the forms $\Theta$ and $\omega$ are given in coordinates they can be defined intrinsically.

2. It can be read off from its coordinate expression that $\Theta$ is the most general 2horizontal $n$-form on $\mathcal{E}$ and that $\mathcal{P}$ can also be identified with the space of all forms of this type.

3. Truncating the translation part by a condition like $p=-\mathcal{H}\left(x^{\mu}, q^{i}, p_{i}^{\mu}\right)$ leeds to a smaller phase space $\tilde{\mathcal{P}}$ of dimension $(N+1)(n+1)-1$. 
The relationships between the various spaces and bundle structures in multisymplectic geometry can be read off the diagram

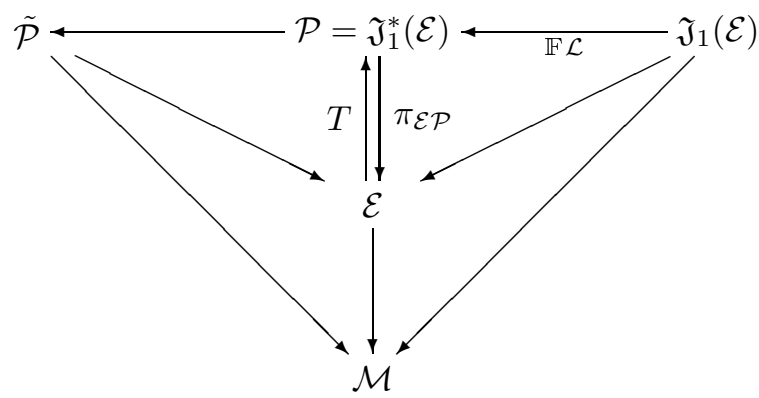

Here $\mathbb{F} \mathcal{L}$ is the fibre derivative associated to the Lagrangean density $\mathcal{L}$. The latter should be interpreted as a function on the first jet bundle to $\mathcal{E}$,

$$
\begin{aligned}
\mathcal{L}: \quad \mathfrak{J}_{1}(\mathcal{E}) & \rightarrow \mathbb{R} \\
\left(x^{\mu}, q^{i}, q_{\mu}^{i}\right) & \mapsto \mathcal{L}\left(x^{\mu}, q^{i}, q_{\mu}^{i}\right) .
\end{aligned}
$$

Then $\mathbb{F} \mathcal{L}$ - which is also known as the covariant Legendre transformation - is given by

$$
\mathbb{F} \mathcal{L}\left(x^{\mu}, q^{i}, q_{\mu}^{i}\right)=\left(x^{\mu}, q^{i}, p_{i}^{\mu}=\frac{\partial \mathcal{L}}{\partial q_{\mu}^{i}}, p=\mathcal{L}-\frac{\partial L}{\partial q^{i} \mu} q_{\mu}^{i}\right) .
$$

The subject of this note is a description of the De Donder-Weyl equations and their properties in terms of general multisymplectic geometry, more precisely in terms of distributions associated to separable Hamiltonean $n$-vectorfields on $\mathcal{P}$ and their integral manifolds.

In contrast to this programme Echeverría-Enríqez et al. ([2]) have worked on the truncated space $\tilde{\mathcal{P}}$, also employed by Kanatchikov $([5,6])$. The price to be payed for this is the necessity to introduce a connection into a fully geometric treatment.

Martin ([9]) has investigated De Donder-Weyl equations on very special multisymplectic manifolds only. Although those cases show very interesting additional properties as, for example, a generalised Darboux theorem ([8]), they only cover the case when $\mathcal{E}$ is some antisymmetrised tensor bundle of the tangent bundle to $\mathcal{M}$.

Let us briefly define the necessary geometric notions. An $r$-vectorfield $X$ on $\mathcal{P}$ is just a totally antisymmetric covariant vectorfield on $\mathcal{P}$, i.e. $X$ is a section of the $r$-th exterior power of the tangent bundle $T \mathcal{P}$,

$$
X \in \Gamma \Lambda^{r} T \mathcal{P} .
$$

An $r$-vectorfield $X$ is called (locally) separable, if there are vectorfields $Z_{1}, \ldots, Z_{r}$ such that (locally)

$$
X=Z_{1} \wedge \cdots \wedge Z_{r}
$$


An $r$-distribution on $\mathcal{P}$ is a smooth collection of $r$-dimensional subspaces of the tangent space $T_{p} \mathcal{P}$ for all $p \in \mathcal{P}$. Taking vectorfields $Z_{1}, \ldots, Z_{r}$ whose values (locally) span the $r$-dimensional subspaces at every point, wee see that distributions can be described by separable $r$-vectorfields ([2, 10], for an introduction into the theory of integrable distributions and foliations see [f]).

An $r$-distribution on $\mathcal{P}$ is called integrable, if locally every point $p$ of $\mathcal{P}$ lies in a unique integral submanifold $\mathcal{N}$ of dimension $r$ of $\mathcal{P}$ such that for $p \in \mathcal{M} T_{p} \mathcal{N}$ is the subspace of $T_{p} \mathcal{P}$ defined by the distribution. A separable $r$-vectorfield defining an integrable distribution will also be called integrable.

An $(n-r)$-form $f$ and an $r$-vectorfield $X_{f}$ are called Hamiltonean, if

$$
d f=i_{X_{f}} \omega .
$$

There are two essential features that do not occur in the case $n=1$ of classical mechanics. Firstly, although $\omega$ is non-degenerate, $X_{f}$ is not uniquely defined by $d f$ for $r \neq 1$.

Moreover, by far not every $(n-r)$-form $f$ is Hamiltonean. There are in general strong constraints both on $f$ and on $X_{f}$ which are satisfied trivially for $n=1$ (in this case, $r=1$ is the only possibility) or $r=n$.

Consider now a function $h\left(x^{\mu}, q^{i}, p_{i}^{\mu}, p\right)$ which automatically gives a Hamiltonean zero form. It turns out that separable Hamiltonean $n$-vectorfields $X_{h}$ on $\mathcal{P}$ with

$$
i_{X_{h}} \omega=d h
$$

can be found provided $h$ depends on $p$ in a particular way, namely

$$
h\left(x^{\mu}, q^{i}, p_{i}^{\mu}, p\right)=-\mathcal{H}\left(x^{\mu}, q^{i}, p_{i}^{\mu}\right)-p,
$$

where $\mathcal{H}$ is a function not depending on $p$.

We shall show that the Hamiltonean $n$-vectorfield $X_{h}$ describes a solution of the De Donder-Weyl equations (5) for $\mathcal{H}$. For the solutions of the De Donder-Weyl equations we shall always have to assume that the projection of the values of $X_{h}$ down to $\mathcal{M}$ has to be of maximal rank. This motivates the ansatz

$$
Z_{\mu}=\frac{\partial}{\partial x^{\mu}}+\left(Z_{\mu}\right)^{i} \frac{\partial}{\partial q^{i}}+\left(Z_{\mu}\right)_{i}^{\nu} \frac{\partial}{\partial p_{i}^{\nu}}+\left(Z_{\mu}\right)_{0} \frac{\partial}{\partial p}, \quad \mu=1, \ldots, n
$$

for the $n$ vectorfields that shall be combined to yield $X_{h}$,

$$
Z_{1} \wedge \cdots \wedge Z_{n} \stackrel{!}{=} X_{h}
$$

Inserting (15) and (16) into (13) gives

$$
\begin{aligned}
\left(Z_{\mu}\right)^{i} & =\frac{\partial}{\partial p_{i}^{\mu}} h \\
\left(Z_{\mu}\right)_{i}^{\mu} & =-\frac{\partial}{\partial q^{2}} h \\
\left(Z_{\mu}\right)_{0} & =-\frac{\partial}{\partial p}+\left(Z_{\mu}\right)^{i} \frac{\partial}{\partial q^{i}} h+\left(Z_{\mu}\right)_{i}^{\nu} \frac{\partial}{\partial p_{i}^{\nu}} h .
\end{aligned}
$$


Equation (18) does not determine $\left(Z_{\mu}\right)_{i}^{\nu}$ completely. This corresponds to the fact that the De Donder-Weyl equations do determine the change of $\varphi^{i}$ along the direction $\frac{\partial}{\partial x^{\mu}}$ uniquely but do not fix all components of the change of the polymomenta. Rather, only the combination $\partial_{\mu} \pi_{i}^{\mu}$ is fixed by the equations.

The general solution of (18) is hence

$$
\left(Z_{\mu}\right)_{i}^{\nu}=-\frac{1}{n} \delta_{\mu}^{\nu} \frac{\partial}{\partial q^{i}} h+\left(Z_{\mu}^{\prime}\right)_{i}^{\nu} \text { with }\left(Z_{\mu}^{\prime}\right)_{i}^{\mu}=0 .
$$

So the vectorfields $Z_{\mu}$ are given by (15), (17), and (20), as the remaining component $\left(Z_{\mu}\right)_{0}$ can be computed from the other ones using (19),

$$
\left(Z_{\mu}\right)_{0}=-\frac{\partial}{\partial p}+\frac{n-1}{n} \frac{\partial}{\partial p_{i}^{\mu}} h \frac{\partial}{\partial q^{i}} h+\frac{\partial}{\partial p_{i}^{\nu}} h\left(Z_{\mu}^{\prime}\right)_{i}^{\nu} .
$$

The following theorem is a corollary of the above observations. A detailed proof can be found in [10].

THEOREM 1.

1. For $h \in \mathcal{C} \infty(\mathcal{P})$ of the form

$$
h\left(x^{\mu}, q^{i}, p_{i}^{\mu}, p\right)=-\mathcal{H}\left(x^{\mu}, q^{i}, p_{i}^{\mu}\right)-p
$$

there exist locally associated separable Hamiltonean n-vectorfields $X_{h}$ on $\mathcal{P}$, whose projection onto the base $\mathcal{M}$ has maximal rank $n$.

2. The integral manifolds of $X_{h}$ (provided they exist) correspond to solutions of the De Donder-Weyl Hamiltonean $\mathcal{H}$.

3. Let $\varphi$ be a (local) solution of the De Donder-Weyl equation (5) with De DonderWeyl Hamiltonean $\mathcal{H}$. Then the tangent spaces along $\varphi$ describe an $n$-distribution and hence a separable n-vectorfield that is Hamiltonean w.r.t. $h$.

Next we shall investigate integrability conditions of the $n$-vectorfield $X_{h}$, which will lead us to a Hamilton-Jacobi formulation of the De Donder-Weyl equations.

First of all, it is important to notice that $X_{h}$ does not project to a vectorfield on the extended configuration space $\mathcal{E}$. This is due to the fact that the solutions of the equations of motion also depend on the initial momenta. Pictorially, this is shown in figure 1 .

Moreover and unlike the situation in classical mechanics, the totality of solutions of the De Donder-Weyl equations does not give rise to a foliation of the multisymplectic phase space. As a matter of fact, there are many solutions through every point $\left(x^{\mu}, q^{i}, p_{i}^{\mu}, p\right)$ of $\mathcal{P}$. In general, one can not even expect the existence of a foliating subset of solutions. A more modest task is the discussion of foliations of the extended configuration space by solutions of the De Donder-Weyl equations projected down from $\mathcal{P}$ to $\mathcal{E}$. In classical mechanics, this is achieved by suitable solutions of the time dependent Hamilton-Jacobi equations

$$
\frac{\partial S(q, t)}{\partial t}+H\left(q, \frac{\partial S}{\partial q}, t\right)=0
$$




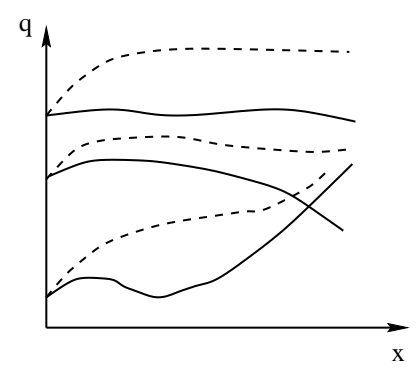

Fig. 1: Projected solutions with different initial momenta.

and

$$
p_{i}=\frac{\partial S(q, t)}{\partial q^{2}}, \quad E=-\frac{\partial S(q, t)}{\partial t} .
$$

Equation (24) defines a map

$$
T: \mathcal{Q} \times \mathbb{R} \rightarrow T^{*} \mathcal{Q} \times \mathbb{R}^{2},
$$

where the relation between $T$ and $S$ is given by

$$
T\left(t, q^{i}\right)=\left(t, q^{i}, T_{i}\left(t, q^{i}\right), T_{0}\left(t, q^{i}\right)\right), \quad T_{i}=\partial_{i} S, \quad T_{0}=\partial_{t} S .
$$

Analogously, we shall consider a map

$$
\begin{aligned}
T: \mathcal{E} & \rightarrow \mathcal{P} \\
e=\left(x^{\mu}, q^{i}\right) & \mapsto T(e)=\left(x^{\mu}, q^{i}, T_{i}^{\mu}(e)=p_{i}^{\mu}, T_{0}(e)=p\right)
\end{aligned}
$$

also in field theory.

For separable $n$-vectorfields $X_{h}=Z_{1} \wedge \cdots Z_{n}$, we can use $T$ and the tangential map of the projection onto $\mathcal{E}, T \pi_{\mathcal{E P}}$, to obtain $n$ vectorfields on $\mathcal{E}$,

$$
\left(\tilde{Z}_{\mu}\right)(e)=T \pi_{\mathcal{E P}} Z_{\mu}(T(e)) .
$$

The $\tilde{Z}_{\mu}$ can be combined to yield a projected distribution

$$
\tilde{X}_{h}=\tilde{Z}_{1} \wedge \cdots \tilde{Z}_{n},
$$

which can be expected to be generically integrable and to give a local foliation of $\mathcal{E}$. As a matter of fact, a generalisation of the flux straightening theorem tells us that a (local) foliation of $\mathcal{E}$ by an $N$ parameter family of solutions of the De Donder-Weyl equation arises iff these solutions can be transformed (locally) into constant solutions by a bundle automorphism $\Phi$ of $\mathcal{E}$ as indicated in figure 2. More precisely, one can prove the following theorem. 


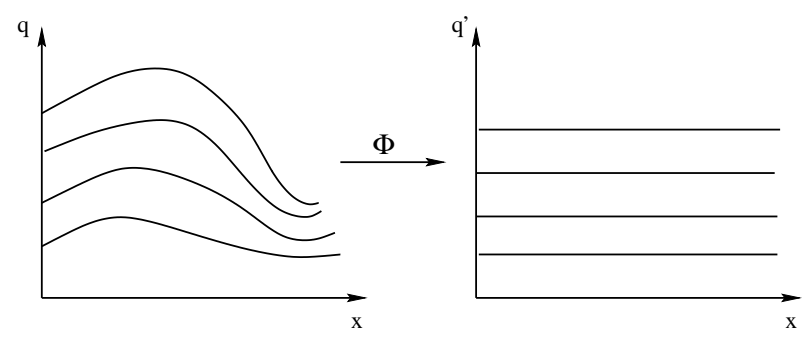

Fig. 2: A foliation yields a trivialisation where the leaves become constant sections.

Theorem 2. Let $\mathcal{H}$ be a regular De Donder-Weyl Hamiltonean on $\mathcal{P}$. Then there exists a local foliation of $\mathcal{E}$ by projected solutions of the De Donder-Weyl equations if and only if there is a (local) section $T: \mathcal{E} \rightarrow \mathcal{P}$ of the bundle $\mathcal{P} \rightarrow \mathcal{E}$, which in suitable bundle coordinates fulfills

$$
\begin{aligned}
\frac{\partial}{\partial x^{\mu}} T_{i}^{\mu}(x, q) & =-\left(\frac{\partial}{\partial q^{2}} \mathcal{H}\right)\left(x, q, T_{i}^{\mu}(x, q)\right) \\
\frac{\partial}{\partial x^{\mu}} T_{0}(x, q) & =-\left(\frac{\partial}{\partial x^{\mu}} H\right)\left(x, q, T_{i}^{\mu}(x, q)\right) \\
\frac{\partial}{\partial x^{\mu}} T_{i}^{\mu}(x, q) & =-\left(\frac{\partial}{\partial q^{2}} T_{0}\right)(x, q) \\
\left(\frac{\partial}{\partial q^{i}} \mathcal{H}\right)\left(x, q, T_{i}^{\mu}(x, q)\right) & =0
\end{aligned}
$$

Here, equations (30) - (32) arise from the De Donder-Weyl equation on $\mathcal{P}$, while condition (33) ensures integrability. A detailed proof can be found in [10]. If a collection of functions $S^{\mu}(x, q), \mu=1, \ldots, n$, solves the generalised Hamilton-Jacobi equation

$$
\frac{\partial}{\partial x^{\mu}} S^{\mu}(x, q)+\mathcal{H}\left(x, q, \frac{\partial}{\partial q^{i}} S^{\mu}(x, q)\right)
$$

then

$$
T_{i}^{\mu}=\frac{\partial}{\partial q^{2}} S^{\mu}, \quad T_{0}=\frac{\partial}{\partial x^{\mu}} S^{\mu}
$$

solve the first three conditions (30) - (32) of theorem 2. Obviously, this constitutes a generalisation of (26).

Equation (33) is the foliation condition of the family of projected solutions. It just means that in suitable bundle coordinates of $\mathcal{E}$ the leaves of the foliation constitute a family of constant fields (sections). The map $T: \mathcal{E} \rightarrow \mathcal{P}$ them arises by a jet prolongation of these fields followed by the fibre derivative (covariant Legendre transformation) $\mathbb{F} \mathcal{L}: \mathfrak{J}_{1}(\mathcal{E}) \rightarrow$ $\mathcal{P}$.

Finally, we will show how (30) - (32) can be cast into an intrinsic geometric form. Defining the horizontal $(n-1)$-form

$$
S=S^{\mu} d^{n} x_{\mu}
$$


and the 2-horizontal $n$-form $d S$ and using the fact that $\mathcal{P}$ can be identified with the space of all 2-horizontal $n$-forms, we can write equation (34) as

$$
h \circ d S=0,
$$

where $h$ is related to $\mathcal{H}$ as in (14). The Cartan-Weyl form $\Theta$ fulfills,

$$
\Theta(d S)=d S
$$

More generally, the conditions $T=d S$ and (37) can be weakened to

$$
d T=0, \quad d(h \circ T)=0,
$$

where $T$ is a (local) 2-horizontal $n$-form $T$ (and hence defines a (local) map from $\mathcal{E}$ to $\mathcal{P})$. The set of equations $(39)$ is equivalent to the coordinate conditions (30) - (32).

\section{REFERENCES}

[1] F. Cantrijn, A. Ibort, M. De Leon: On the geometry of multisymplectic manifolds. J. Aust. Math. Soc., Ser. A 66, No.3 (1999), 303-330.

[2] A. Echeverría-Enríquez, M. C. MuÑoz-Lechnda, N. Román-Roy: Multivectorfield Formulation of Hamiltonian Field Theories: Equations and Symmetries. J. Phys. A: Math. Gen. 32 (1999), 8461-8484.

[3] M. Forger, H. Römer: A Poisson Bracket on Multisymplectic Phase Space., to appear in the proceedings of the 32nd Symposium on Mathematical Physics, Torun, Poland, June 2000 , math-ph/0009037.

[4] M. J. Gotay, J. Isenberg, and J. E. Marsden: Momentum Maps and Classical Relativistic Fields I: Covariant Field Theory, physics/9801019.

[5] I. V. Kanatchikov: Rep. on Math. Phys. 40.2 (1997), 225-234, hep-th/9710069.

[6] I. V. Kanatchikov: Rep. on Math. Phys. 41.1 (1998), 49-90, hep-th/9709229.

[7] I. Koláŕ, P. W. Michor, and J. Slovák: Natural Operations in Differential Geometry. Springer-Verlag, Berlin, Heidelberg, New York, 1993.

[8] G. Martin: A Darboux Theorem for Multi-Symplectic Manifolds. Lett. Math. Phys. 16 (1988), 133-138.

[9] G. Martin: Dynamical structures for k-vector fields. Int. J. Theor. Phys. 27, No.5 (1988), 571-585.

[10] C. Paufler, H. Römer: Geometry of Hamiltonian n-vectors in Multisymplectic Field Theory., math-ph/0102008,

[11] D. J. Saunders: The Geometry of Jet Bundles. Lond. Math. Soc. Lect. Note Ser. 142, Cambr. Univ. Pr., Cambridge, 1989. 